Check for updates

Cite this: J. Mater. Chem. A, 2020, 8 , 2490

Received 21st November 2019 Accepted 25th December 2019

DOI: $10.1039 / c 9 t a 12768 b$

rsc.li/materials-a

\section{Promoting the hydrogen evolution reaction through oxygen vacancies and phase transformation engineering on layered double hydroxide nanosheets $\uparrow$}

Shujie Liu, ${ }^{a}$ Jie Zhu, ${ }^{a}$ Mao Sun, ${ }^{a}$ Zhixue Ma, ${ }^{a}$ Kan Hu, ${ }^{a}$ Tomohiko Nakajima, (D) *b Xianhu Liu, (D) ${ }^{c}$ Patrik Schmuki (D) ${ }^{* d}$ and Lei Wang (D)*ac

In electrocatalysis, layered double hydroxide (LDH) materials have attracted considerable interest in promoting the oxygen evolution reaction (OER). For the hydrogen evolution reaction (HER), these materials show a poor electrocatalytic activity. In this work, we take CoFe LDH grown on nickel foam as a model system, and for the first time we treat CoFe LDH under plasma etching conditions, which results in a highly active HER electrocatalyst in alkaline media. Massive defects and amorphous phase formation occur during plasma etching. More importantly, phase transformation of CoFe LDH into $\mathrm{CoFe}_{2} \mathrm{O}_{4}$ takes place. Additional introduction of Ce leads to more active sites and improved electrical conductivity, which provide an overpotential of $73 \mathrm{mV}$ at a current density of $10 \mathrm{~mA} \mathrm{~cm}{ }^{-2}$ for V-Ce/CoFe LDH. The experimental results match well with DFT calculations, proving that defect engineering, phase transformation, and electronic structure tailoring can result in a high efficiency of HER activity. Moreover, a cell constructed of CoFe LDH\|V-Ce/CoFe LDH is capable of overall water splitting with a cell voltage as low as $1.65 \mathrm{~V}$ at $10 \mathrm{~mA} \mathrm{~cm}{ }^{-2}$ and a remarkable long-term stability of $60 \mathrm{~h}$. The plasma etching strategy can also be effective for other LDH-based electrocatalysts (e.g. NiFe LDH and NiCo LDH), which demonstrates the universality of the strategy for improving HER performance. This work provides a simple but feasible pathway for designing alkaline HER electrocatalysts based on advanced LDH materials.

\section{Introduction}

Electrochemical water splitting, consisting of the oxygen evolution reaction (OER) and the hydrogen evolution reaction (HER), has been explored and used for decades. Noble metals are so far the most efficient electrocatalyst (e.g. iridium dioxide/ ruthenium dioxide for the OER, and platinum for the HER) owing to their low overpotential and fast kinetics. ${ }^{1-5}$ However, the high cost usually limits their large-scale applications. Therefore, it is necessary and urgent to design new efficient

${ }^{a}$ School of Chemistry and Chemical Engineering, Inner Mongolia Engineering and Technology Research Center for Catalytic Conversion and Utilization of Carbon Resource Molecules, Inner Mongolia University, 235 West University Street, Hohhot 010021, P. R. China. E-mail: wanglei@imu.edu.cn

${ }^{b}$ Advanced Coating Technology Research Center, National Institute of Advanced Industrial Science and Technology, Tsukuba Central 5, 1-1-1 Higashi, Tsukuba, Ibaraki 305-8565, Japan. E-mail: t-nakajima@aist.go.jp

'Key Laboratory of Materials Processing and Mold, Ministry of Education, Zhengzhou University, Zhengzhou 450002, P. R. China

${ }^{d}$ Department of Materials Science and Engineering WW4-LKO, University of ErlangenNuremberg, Martensstrasse 7, D-91058 Erlangen, Germany. E-mail: schmuki@ww. uni-erlangen.de

$\dagger$ Electronic supplementary information (ESI) available. See DOI: $10.1039 / \mathrm{c} 9 \operatorname{ta} 12768 \mathrm{~b}$ electrocatalysts with low cost and high earth abundance, instead of noble metal-based catalysts. Moreover, in the present 2-compartment electrolyzer cells, an optimized anode in a suitable anode electrolyte and an optimized cathode in a suitable cathode electrolyte are operated, e.g. in a membrane configuration. Operation in a one-electrolyte configuration would require the presence of a catalyst that is able provide HER and OER activity. ${ }^{6,7}$

In this context, recently, layered double hydroxide materials (e.g. NiFe $\mathrm{LDH}, \mathrm{CoFe} \mathrm{LDH}$, and NiCo LDH) have attracted intensive interest towards the OER. ${ }^{8-13}$ However, the HER electrocatalytic activity is unsatisfactory, due to the poor conductivity and insufficient electrochemical active sites for this reaction. Oxygen vacancy engineering has been applied to promote OER and HER performances, owing to its beneficial effects on charge transfer and dissociation of water molecules. ${ }^{13,14}$ The absence of oxygen ions changes the electronic structure of the surface and thus transforms a semiconductor into a metal-like conductor, combined with dangling bonds and gap states. Another effect is the generation of low coordination metals that leads to active sites of metal oxides/hydroxides. ${ }^{1}$ Besides, altering the nature and density of oxygen vacancies in LDH materials exposes use of surface atoms. ${ }^{15-17}$ For example, 
Wang and coworkers reported that exfoliation of bulk CoFe LDH into ultrathin LDH nanosheets through Ar/water plasma etching resulted in the formation of multiple vacancies, including $\mathrm{O}$, Co, and Fe vacancies, for improving the intrinsic OER activity. ${ }^{15,16}$ According to these treatments, the enhanced OER performances can be achieved for LDH-based catalysts in alkaline media. However, these electrocatalysts are still inert for the HER, let alone for overall water splitting. Therefore, it is still a challenge to achieve highly efficient HER activity of LDHbased materials, for developing bifunctional OER and HER electrocatalysts for overall one-pot water splitting.

We first perform a density functional theory (DFT) calculation to determine if the oxygen vacancies can influence the electronic structure of LDH and the surface adsorption energy of the reactants, and further the HER activity. Taking CoFe LDH material as an example, oxygen vacancies are constructed and Ce doping is considered and simulated by replacing $\mathrm{O}$ on the top surface. The optimized structures are shown in Fig. 1a. The HER activity is governed by the adsorption free energy of hydrogen $\left(\Delta G_{\mathrm{H}^{*}}\right) .^{\mathbf{1}, \mathbf{1 8}}$ For a fast proton/electron-transfer process and hydrogen release process, the $\Delta G_{\mathrm{H}^{*}}$ value should be close to zero. Fig. 1b shows that the $\left|\Delta G_{\mathrm{H}^{*}}\right|$ of CoFe LDH with oxygen vacancies (V-CoFe $\mathrm{LDH}$ ) is $0.18 \mathrm{eV}$, which is much lower than that of $\mathrm{CoFe} \mathrm{LDH}(0.55 \mathrm{eV})$. This indicates favorable $\mathrm{H}^{*}$ adsorption kinetics on V-CoFe LDH for the HER. Besides, Ce insertion into $\mathrm{CoFe} \mathrm{LDH}(\mathrm{Ce} / \mathrm{CoFe} \mathrm{LDH})$ exhibits a $\left|\Delta G_{\mathrm{H}^{*}}\right|$ value of $0.26 \mathrm{eV}$. Further oxygen vacancies provide a $\left|\Delta G_{\mathrm{H}^{*}}\right|$ value of $0.14 \mathrm{eV}$ for $\mathrm{V}-\mathrm{Ce} / \mathrm{CoFe} \mathrm{LDH}$. This confirms that Ce doping and oxygen vacancies indeed can lower the energy barrier and facilitate the HER process, as well as enhance the HER activity.

Motivated by the promising predictions, in this work, we synthesize CoFe LDH with oxygen vacancy defects by a plasma etching strategy and establish it as a highly active HER catalyst in alkaline media. In contrast to the oxygen vacancy engineering, the phase transformation of CoFe LDH into $\mathrm{CoFe}_{2} \mathrm{O}_{4}$ occurs. Further, introduction of Ce leads to more active sites and improved electrical conductivity, resulting in a high efficiency of HER activity for V-Ce/CoFe LDH. The experimental results agree well with the DFT calculation, proving that the oxygen vacancy defects in $\mathrm{CoFe} \mathrm{LDH}$ or $\mathrm{Ce} / \mathrm{CoFe} \mathrm{LDH}$ lead to an outstanding HER performance. More importantly, the CoFe $\mathrm{LDH} \| \mathrm{V}-\mathrm{Ce} / \mathrm{CoFe} \mathrm{LDH}$ is capable of overall water splitting with a cell voltage as low as $1.65 \mathrm{~V}$ at a current density of $10 \mathrm{~mA} \mathrm{~cm}^{-2}$

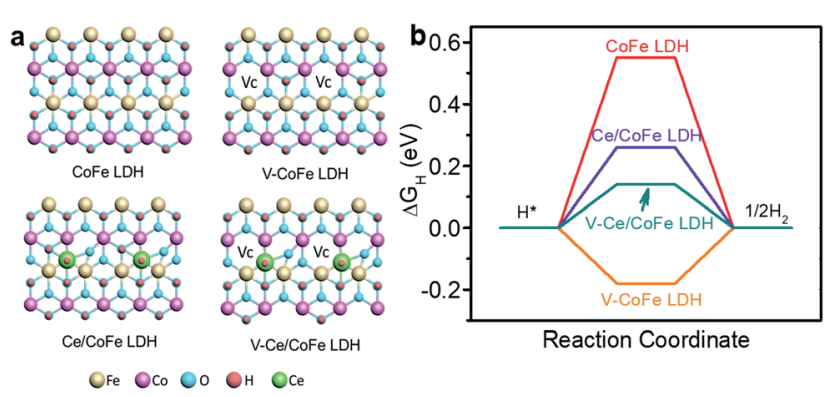

Fig. 1 (a) Structures and (b) free energy diagram of the HER over CoFe LDH, V-CoFe LDH, Ce/CoFe LDH, and V-Ce/CoFe LDH. and remarkable long-term stability. These findings provide a simple but feasible pathway for the design of advanced LDH materials for energy conversion devices.

\section{Results and discussion}

Fig. 2a illustrates the synthesis of $\mathrm{V}$-CoFe $\mathrm{LDH}$ and $\mathrm{V}-\mathrm{Ce} / \mathrm{CoFe}$ LDH. First, CoFe LDH is prepared by a hydrothermal method in an aqueous solution containing $\mathrm{Co}\left(\mathrm{NO}_{3}\right)_{2} \cdot 6 \mathrm{H}_{2} \mathrm{O}$ and $\mathrm{Fe}\left(\mathrm{NO}_{3}\right)_{3} \cdot 9 \mathrm{H}_{2} \mathrm{O}$ with a molar ratio of $\mathrm{Co}: \mathrm{Fe}=1: 1$. The CoFe LDH is composed of 2 dimensional (2D) nanosheets with a thickness of 40-60 nm (Fig. S1a and S2 $\dagger$ ). Transmission electron microscopy (TEM) images (Fig. S3†) present the lattice fringes with distances of $0.26 \mathrm{~nm}, 0.23 \mathrm{~nm}$, and $0.15 \mathrm{~nm}$, matching well with the (012), (015), and (110) planes of CoFe LDH, respectively. Subsequently, the CoFe LDH was subjected to plasma etching to synthesize the defected CoFe LDH (denoted as V-CoFe LDH). As shown in Fig. S1b, $\uparrow$ many pores and small fragments are observed. TEM images present a nanosheet structure consisting of small nanocrystallites, and these small nanocrystallites are surrounded by amorphous shells (Fig. S4 $\dagger$ ). Atomic force microscopy (AFM) images show that the height of $\mathrm{V}$-CoFe LDH is approximately $5 \mathrm{~nm}$, which is around 10 times thinner compared to a single layer of $\mathrm{LDH}$ (Fig. S2 $\dagger$ ). This indicates that $\mathrm{LDH}$ could be exfoliated, in line with the results reported by Wang et al. ${ }^{17}$ We further induced Ce doping into the LDH as shown in Fig. 2a. No obvious change in morphology is observed compared to the CoFe LDH (Fig. 2b, S1c, and S5 $\dagger$ ). Further $\mathrm{N}_{2}$ plasma treatment leads to abundant amorphous sites and defects detected in V-Ce/CoFe LDH (Fig. 2h and S6 $\dagger$ ), which are similar to the V-CoFe LDH. The elemental mapping images from energy dispersive X-ray spectroscopy (EDS) confirm a homogeneous distribution of $\mathrm{Co}, \mathrm{Fe}, \mathrm{Ce}$, and $\mathrm{O}$ species (Fig. 2j). Closely resembling the $\mathrm{V}$-CoFe $\mathrm{LDH}$, the thickness of $\mathrm{V}$ $\mathrm{Ce} / \mathrm{CoFe} \mathrm{LDH}$ nanosheets decreases to $\sim 5 \mathrm{~nm}$ after the etching process (Fig. S7†). Combined with the TEM results, abundant defects and amorphous phases caused by the plasma etching might induce a higher surface area and more active sites, facilitating the electron transfer and $\mathrm{OH}^{-} / \mathrm{H}_{2} \mathrm{O}$ adsorption, subsequently further promoting the HER activity.

The crystalline structures of CoFe LDH and Ce/CoFe LDH without and with $\mathrm{N}_{2}$ plasma etching were investigated by X-ray diffraction (XRD). The corresponding powders (Fig. S8 $\dagger$ ) were examined, as the low amount of LDHs growing on nickel foam (NF) led to weak signals in XRD spectra. Fig. 3a shows that two phases composed of CoFe LDH (JCPDS 50-0235) and $\mathrm{CoFe}_{2} \mathrm{O}_{4}$ (JCPDS 01-1121) exist in the LDH powders. ${ }^{19}$ Through $\mathrm{N}_{2}$ plasma etching treatment, all diffraction peaks that can be assigned to (00l) ( $l=3,6$ and 9), (015) and (018) in LDH decrease, while the $\mathrm{CoFe}_{2} \mathrm{O}_{4}$ remains unaffected by it. The different plasma etching times of XRD patterns were further investigated as shown in Fig. S9a. $\dagger$ With increasing etching time, the diffraction peaks for the LDH finally disappear after $120 \mathrm{~s}$. To probe the details of phase transformation, 2D XRD mappings were conducted as shown in Fig. S9b. $\dagger$ The $(00 l)$ Debye-rings are clearly observed but disappear after $120 \mathrm{~s}$, indicating that this structural transformation of $\mathrm{LDH}$ does not derive from a deformation of crystal 

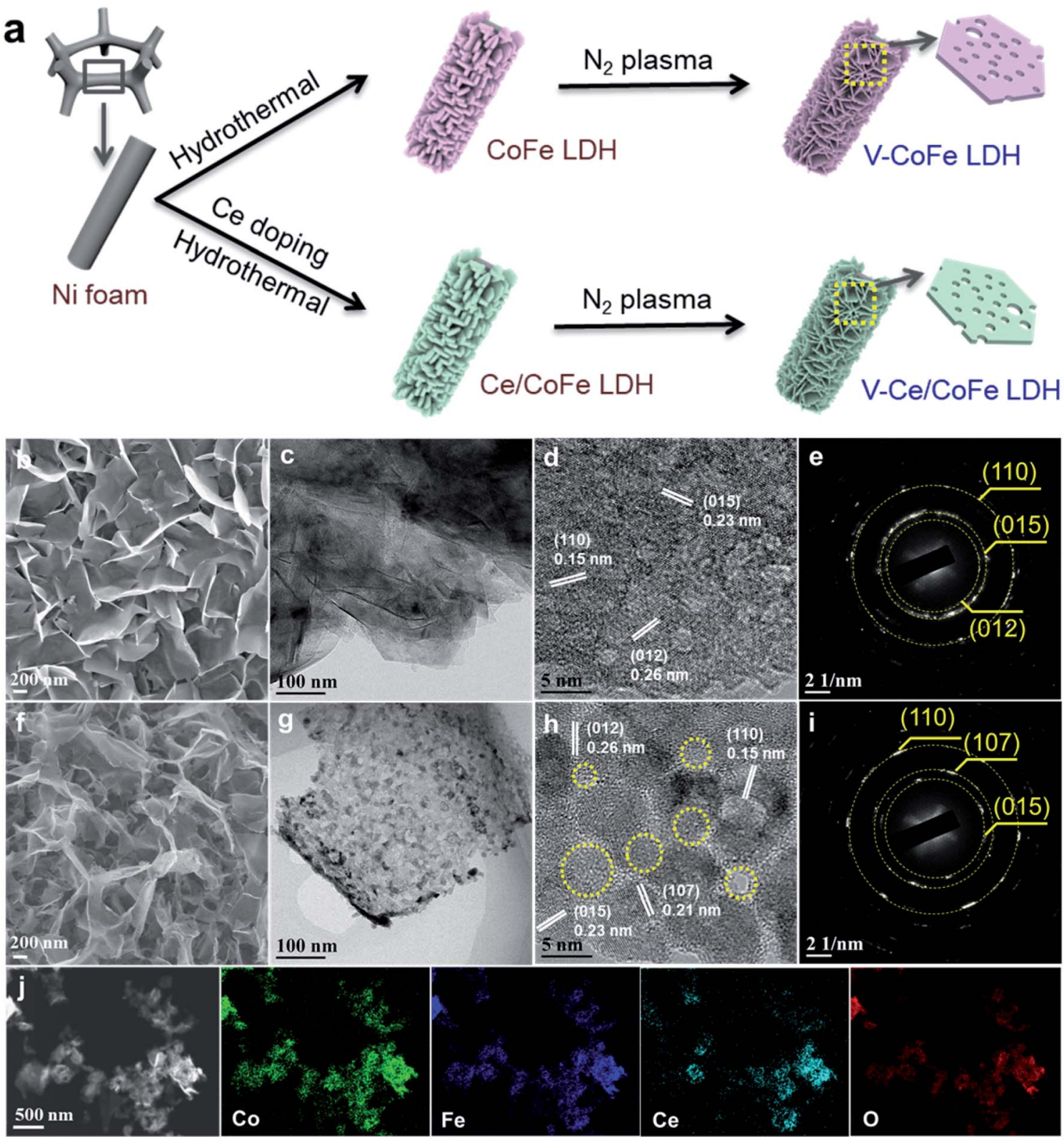

Fig. 2 (a) Schematic illustration of synthesis of V-CoFe LDH and V-Ce/CoFe LDH; (b) SEM image, (c and d) TEM images, and (e) SAED pattern of $\mathrm{Ce} / \mathrm{CoFe}$ LDH; (f) SEM image, (g and h) TEM images, and (i) SAED pattern of $\mathrm{V}$-Ce/CoFe LDH; (j) TEM-EDS mapping images of $V$-Ce/CoFe LDH.

orientation but from the shortening crystal coherent length. By using the collected $\mathrm{X}$-ray diffraction of $2 \mathrm{D}$ mapping in the range of $\beta=-22^{\circ}$ to $22^{\circ}$ ( $\beta$ : Debye-ring direction) for the $\mathrm{LDH}$, we evaluate the lattice strain of $\mathrm{LDH}$ crystals based on WilliamHall analysis (Fig. S9c and $d \dagger$ ). The lattice strain $(\varepsilon)$ can be evaluated using the slope of a plot of $F_{h k l} \cos \theta$ versus $4 \sin \theta$ based on the following equation: ${ }^{20}$

$$
F_{h k l} \cos \theta / \lambda=k \lambda / D+4 \varepsilon \sin \theta
$$

where $F_{h k l}$ is the full width at half-maximum intensity of diffraction peaks, $\theta$ is the diffraction angle, $\lambda$ is the X-ray wavelength (CuK $\alpha$ : $1.5405 \AA$ ), $D$ is the crystallite size, and $k$ is Scherrer's constant (0.94). Both CoFe- and Ce/CoFe LDHs tend to increase the lattice strain with increasing plasma etching time, and the larger strain is introduced in the Ce/CoFe LDH compared to the CoFe LDH owing to Ce doping. This could be also related to the fragmented crystallite sizes of CoFe and Ce/ CoFe LDHs (Fig. S4 and S6†). Different from previous studies, ${ }^{15-17}$ except for the formation of defects, phase transformation is possibly one of the reasons for the adsorption of molecular $\mathrm{H}_{2} \mathrm{O}$ and the enabled water splitting reaction.

$\mathrm{X}$-ray photoelectron spectroscopy (XPS) measurements were performed to probe chemical valence states in the samples. In the Co 2p spectra (Fig. S10 and S11 $\dagger$ ), the peaks at $780.9 \mathrm{eV}$ and $782.0 \mathrm{eV}$ are ascribed to the $\mathrm{Co}^{3+}$ and $\mathrm{Co}^{2+}$. The $\mathrm{Co}^{3+}$ is partially reduced to $\mathrm{Co}^{2+}$, producing oxygen vacancies after plasma etching. The Fe 2 p spectrum shows two peaks at $713.9 \mathrm{eV}$ and 

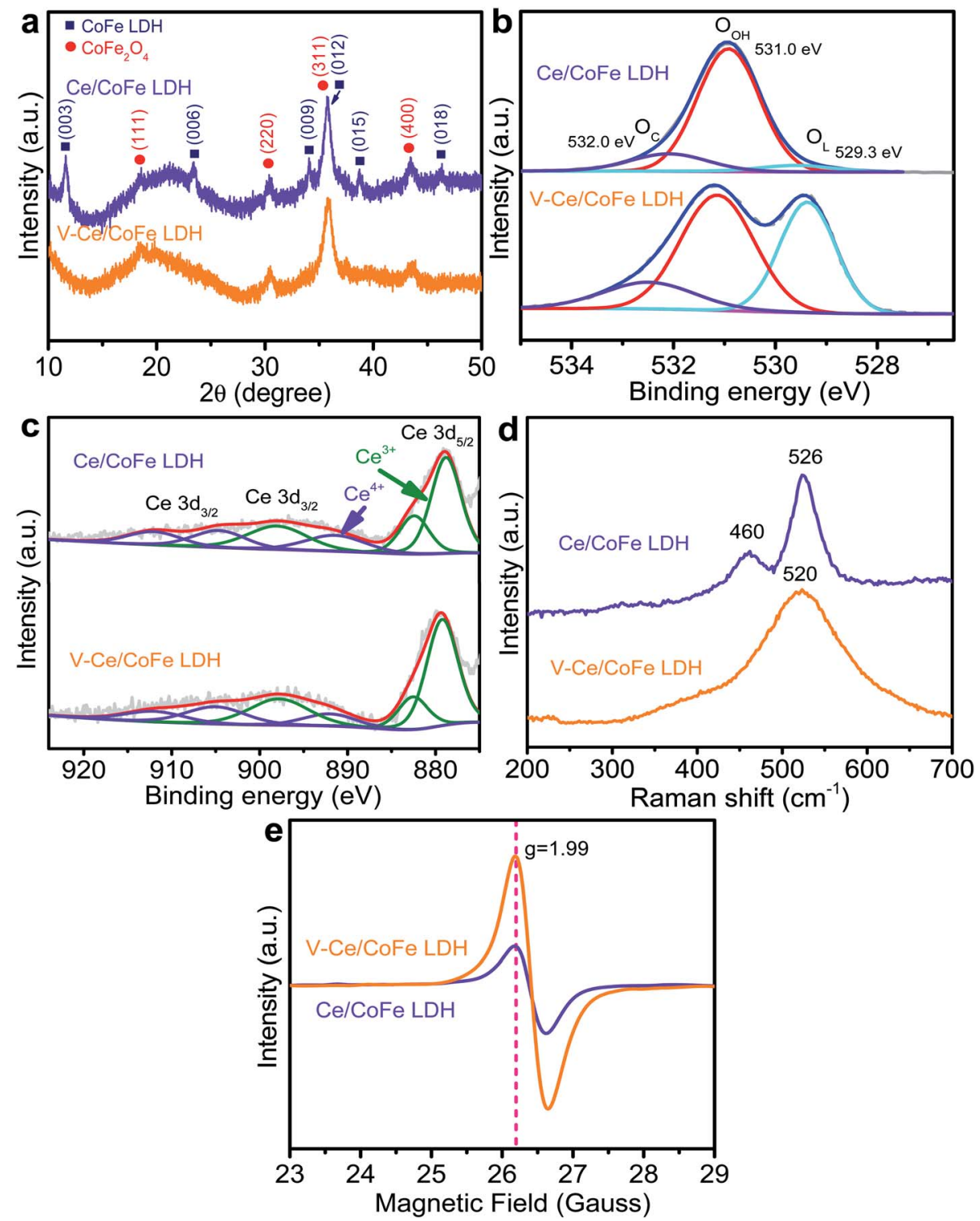

Fig. 3 (a) XRD patterns of Ce/CoFe LDH and V-Ce/CoFe LDH powders; (b) O 1s and (c) Ce 3d XPS spectra, (d) Raman spectra, and (e) EPR results of $\mathrm{Ce} / \mathrm{CoFe} \mathrm{LDH}$ and $\mathrm{V}$-Ce/CoFe LDH.

$724.1 \mathrm{eV}$, indicating the presence of $\mathrm{Fe}^{3+}$ in the CoFe LDH. Compared to the pristine $\mathrm{LDH}$, a new peak around $707.2 \mathrm{eV}$ in Fe 2p of V-CoFe LDH (and V-Ce/CoFe LDH) appears (Fig. S10b and S11b广), which can be ascribed to metallic Fe. Additionally, there is an obvious peak shift toward low binding energies after the plasma treatment. This suggests that the electronic structure in LDH becomes electron-enriched after etching. ${ }^{13,21}$ In Fig. $3 \mathrm{~b}$, the $\mathrm{O} 1 \mathrm{~s}$ peak can be attributed to the metal-oxygen $(\mathrm{M}-\mathrm{O})$ bond in the lattice $(529.3 \mathrm{eV})$, hydroxide bond $(\mathrm{O}-\mathrm{H})$ in the material $(531.0 \mathrm{eV})$, and chemisorbed oxygen $(\mathrm{O}-\mathrm{O})$ on and within the surface $(532.0 \mathrm{eV}){ }^{15}$ There is a negligible change without or with plasma treatment in LDH (Fig. 3b and S10†). However, a remarkable difference is detected for both CoFe- and $\mathrm{Ce} / \mathrm{CoFe}$ LDHs after plasma etching (Fig. S10c and S11c †). Namely, the $\mathrm{O}-\mathrm{H}$ bond has a minute change before and after plasma treatment, while the intensities of $\mathrm{M}-\mathrm{O}$ and absorbed
$\mathrm{O}-\mathrm{O}$ signals are increased after plasma etching. Liu et al. ${ }^{21}$ demonstrated that a LDH layer with tensile strain induced by ball-milling facilitated oxygenated intermediate adsorption. In our case, the increased $\mathrm{O}-\mathrm{O}$ signal indicates an enhanced binding strength of the plasma-etched LDH surface to oxygenated intermediates..$^{13,22,23}$ The $\mathrm{M}-\mathrm{O}$ bond can be ascribed to the transformation of metal-hydroxide into metal oxy-hydroxide. Additionally, the coexistence of $\mathrm{Ce}^{3+}$ and $\mathrm{Ce}^{4+}$ is evidenced in the Ce 3d XPS spectra (Fig. 3c).

Raman spectroscopy was further used to investigate plane vibration modes of corresponding materials, and it reveals a clear difference for samples without and with plasma etching treatments. As shown in Fig. 3d and $\mathrm{S} 12, \uparrow$ the bands at $460 \mathrm{~cm}^{-1}$ and $526 \mathrm{~cm}^{-1}$ attributed to the metal-OH translation mode change to a broadened peak at $516-520 \mathrm{~cm}^{-1}$ after exfoliation, accompanied by a red shift, resulting from phonon 
softening induced by tensile strain..$^{21,24,25}$ Based on $\mathrm{d}$ band theory, lattice strain is able to change the surface $d$ bandwidth, which moves up or down the $\mathrm{d}$ band center to maintain a constant band filling state. ${ }^{21}$ The increased lattice strain in CoFe LDH is expected to upshift the d band center towards the Fermi level, leading to a less filled anti-bonding state and narrow band gap. Mott-Schottky measurements were carried out to determine the flat-band potential $\left(E_{\mathrm{fb}}\right)$ of CoFe LDH. It demonstrates that the $E_{\mathrm{fb}}$ of V-CoFe $\mathrm{LDH}$ is shifted from CoFe LDHs (Fig. S13†), suggesting a better electron conductivity and a high intrinsic HER activity of plasma treated CoFe LDH. Furthermore, electron paramagnetic resonance (EPR) spectroscopy (Fig. 3e and $\mathrm{S} 14 \dagger$ ) demonstrates that the V-CoFe LDH and $\mathrm{V}-\mathrm{Ce} / \mathrm{CoFe} \mathrm{LDH}$ have more defects in the structure, and the signal $(g=1.99)$ reveals structural defects from oxygen vacancies, which is in accordance with a report of Zhao et al. ${ }^{13}$

Fig. 4a shows the linear sweep voltammetry (LSV) results of the corresponding electrocatalysts in $1 \mathrm{M} \mathrm{KOH}$ for their electrocatalytic HER activities. Various plasma power densities, plasma times, and Ce contents were optimized with respect to the best HER performance (Fig. S15 and S16 $\dagger$ ). In Fig. 4a, the $I R$ corrected polarization curve of the V-CoFe LDH exhibits a lower overpotential compared to the pristine CoFe LDH. The optimum overpotential at $10 \mathrm{~mA} \mathrm{~cm}^{-2}$ is $95 \mathrm{mV}$ lower than that of the pristine one (194 mV), indicating a significantly improved HER performance for plasma etched samples. Ce/CoFe LDH displays an overpotential of $156 \mathrm{mV}$ at $10 \mathrm{~mA} \mathrm{~cm}^{-2}$. When the $\mathrm{Ce} / \mathrm{CoFe} \mathrm{LDH}$ was treated in $\mathrm{N}_{2}$ plasma, the overpotential further decreases to $73 \mathrm{mV}$ at $10 \mathrm{~mA} \mathrm{~cm}{ }^{-2}$, close to the $\mathrm{Pt} / \mathrm{C}$ electrode $(30 \mathrm{mV})$, which is one of the best results for the LDH-based electrocatalysts. Moreover, a much smaller slope $\left(69 \mathrm{mV} \mathrm{dec}{ }^{-1}\right.$ ) of the Tafel plot is obtained for the $\mathrm{V}-\mathrm{Ce} / \mathrm{CoFe}$ LDH (Fig. 4b), suggesting an enhanced HER activity due to Ce doping, oxygen vacancies, and phase transformation. These results match well with the DFT calculation as shown in Fig. 1 demonstrating a fast hydrogen release process to facilitate the
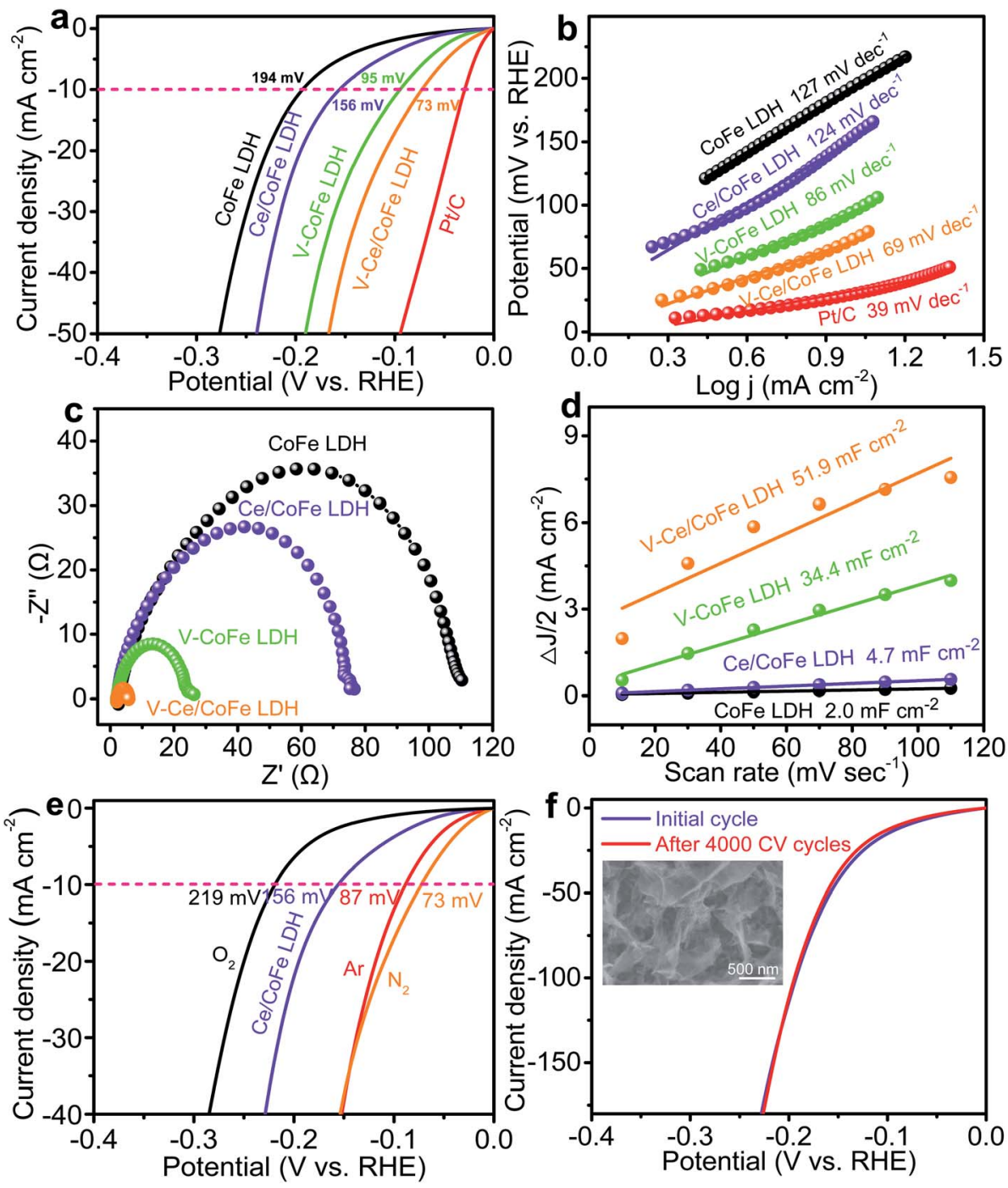

Fig. 4 HER performances of CoFe LDH, V-CoFe LDH, Ce/CoFe LDH, and V-Ce/CoFe LDH in $1 \mathrm{M} \mathrm{KOH}$ electrolyte: (a) IR-corrected polarization curves; (b) Tafel plots; (c) EIS; (d) capacitance current as a function of scan rate; (e) IR-corrected polarization curves of Ce/CoFe LDH plasma treated in various atmospheres; (f) IR-corrected polarization curves of $V$-Ce/CoFe LDH initially and after $4000 \mathrm{CV}$ cycles. 
HER process for the $\mathrm{LDH}$ layer after doping and plasma treatments.

To figure out the nickel foam effect and confirm the advantage of plasma etching treatment, Ce/CoFe $\mathrm{LDH}$ powder was prepared on carbon cloth for comparison. The V-Ce/CoFe LDH powder on carbon cloth has a lower overpotential than the pristine $\mathrm{Ce} / \mathrm{CoFe} \mathrm{LDH}$ powder (Fig. S17†), providing a strong evidence that the plasma etching strategy is indeed beneficial for promoting the HER performance. Additionally, it indicates that the existence of $\mathrm{CoFe}_{2} \mathrm{O}_{4}$ has a positive effect on HER activity.

Electrochemical impedance spectroscopy (EIS) was carried out to obtain more insights into the electron-transfer kinetics of the HER. Fig. 4c shows that V-Ce/CoFe LDH has the smallest charge transfer resistance than other electrodes, implying favorable reaction kinetics. To explore the intrinsic catalytic activities of these catalysts, an electrochemical double-layer capacitance $\left(C_{\mathrm{dl}}\right)$ approach was applied to estimate the electrocatalytic active surface area (ECSA) from cyclic voltammetry curves at various scan rates over a small potential range (Fig. S18 $\dagger$ ). Notably, the V-Ce/CoFe LDH electrode possesses the largest $C_{\mathrm{dl}}$ of $51.9 \mathrm{mF} \mathrm{cm}{ }^{-2}$ compared to those of V-CoFe LDH (34.4 $\left.\mathrm{mF} \mathrm{cm}^{-2}\right)$, Ce/CoFe LDH $\left(4.7 \mathrm{mF} \mathrm{cm}^{-2}\right.$ ), and CoFe LDH (2.0 $\mathrm{mF} \mathrm{cm}^{-2}$ ) shown in Fig. 4d, showing indeed that a larger ECSA of V-Ce/CoFe LDH allows more exposed active sites to promote HER performance.

To rule out any direct plasma atmospheric effect on HER activity, Ce/CoFe LDH samples were treated in different plasma atmospheres (e.g. Ar and $\mathrm{O}_{2}$ ), and the corresponding LSV curves are shown in Fig. 4e. Clearly, the CoFe LDH treated in $\mathrm{Ar}$ or $\mathrm{N}_{2}$ plasma exhibits the improved HER activity, and even close values of overpotentials of $87 \mathrm{mV}$ and $73 \mathrm{mV}$ at $10 \mathrm{~mA} \mathrm{~cm}{ }^{-2}$, respectively, while the sample treated in an oxygen atmosphere displays an increased overpotential $(219 \mathrm{mV})$. XPS shows a very weak N 1s peak (Fig. S19†) due to the short nitrogen plasma time (at $500 \mathrm{~W}$ for $10 \mathrm{~s}$ ). $\mathrm{N}$ is possibly absorbed only on the top surface, so we exclude the effect of $\mathrm{N}$ doping in the LDH, but the oxygen effect points again to oxygen vacancies as the most important factor. We also measured the HER activity in neutral media (Fig. S20 †). Clearly, the V-Ce/CoFe LDH exhibits the best HER performance $\left(212 \mathrm{mV}\right.$ at $\left.10 \mathrm{~mA} \mathrm{~cm} \mathrm{~cm}^{-2}\right)$ among them.
Besides, a thick CoFe LDH material on nickel foam was prepared according to reported literature (Fig. S21 ) $^{26}$ and then treated under the same plasma conditions; the overpotential is reduced from $208 \mathrm{mV}$ (pristine) to $170 \mathrm{mV}$ (Fig. S22 $\dagger$ ). This further demonstrates that the plasma etching does promote the HER activity. However, this thick CoFe LDH did not have a good long-term stability owing to dissolution of $\mathrm{LDH}$ in the electrolyte. Additionally, a thick layer is more easily etched off during the plasma process, which leads to a relatively lower HER performance compared to the LDH material we used in this work. Thus, we choose a thin CoFe LDH layer with a strong adhesion to the substrate for an excellent electrocatalytic performance.

To identify if the plasma etching approach can be applied for other LDH materials, NiFe- and NiCo LDHs were grown on nickel foams and then treated under the same conditions. They also exhibit the enhanced HER performances after plasma etching (Fig. S23†). Furthermore, the V-Ce/CoFe LDH was subjected to CV measurements for 4000 cycles, and the polarization curve has only a tiny change (Fig. 4f), suggesting a good longterm stability. No significant changes in morphology in SEM (inset of Fig. 4f) and TEM images (Fig. S24†) are observed after $\mathrm{CV}$, and the single nanosheet and defects still exist, further confirming a good durability. In the XPS spectra (Fig. S25†), Co, $\mathrm{Ni}$, and Ce are detected, while the $\mathrm{O} 1$ s peak after cycling can be mainly attributed to the hydroxide bond $(\mathrm{M}-\mathrm{OH})$, indicating that the structure has changed from metal oxy-hydroxides to metal hydroxides after the HER. This phenomenon has also been observed in other studies. ${ }^{27,28}$ Based on the results above, in our case, the HER performance of $\mathrm{V}-\mathrm{Ce} / \mathrm{CoFe} \mathrm{LDH}$ is better than or comparable to that of reported non-noble CoFe-based electrocatalysts (Table S1 $\dagger$ ). Therefore, this strategy clearly has a positive effect on the improvement of the HER electrocatalytic activity of LDH-based catalysts.

Finally, inspired by the superior HER performance of the V$\mathrm{Ce} / \mathrm{CoFe} \mathrm{LDH}$ in alkaline media, we used the optimized CoFe $\mathrm{LDH} / \mathrm{V}-\mathrm{Ce} / \mathrm{CoFe} \mathrm{LDH}$ as the anode and cathode to construct a two-electrode electrolyzer for overall water splitting in a $\mathrm{KOH}$ electrolyte. The polarization curve in Fig. 5a shows that the $\mathrm{CoFe} \mathrm{LDH} \| \mathrm{V}-\mathrm{Ce} / \mathrm{CoFe} \mathrm{LDH}$ cell reaches a current density of 10 $\mathrm{mA} \mathrm{cm} \mathrm{cm}^{-2}$ at an overpotential of $1.65 \mathrm{~V}$. Such a performance is
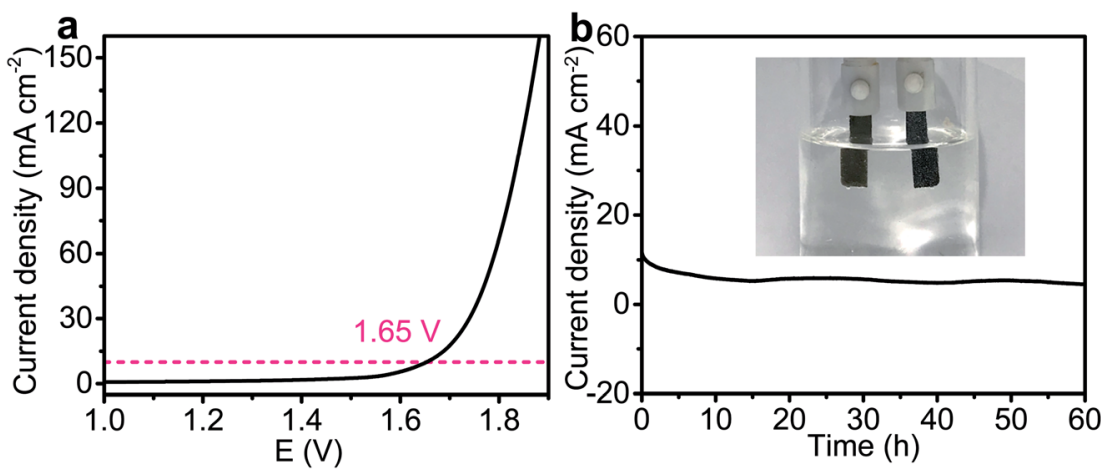

Fig. 5 (a) Performance of the full water-splitting device using the CoFe LDH as the anode and V-Ce/CoFe LDH as the cathode; (b) chronoamperometric curve of CoFe LDH\|V-Ce/CoFe LDH for full water splitting with an overpotential of $10 \mathrm{~mA} \mathrm{~cm} \mathrm{cor}^{-2} 60 \mathrm{~h}$. The inset of b shows the optical photograph of generation gases. 
better than the values reported for CoFe-based electrocatalysts. Furthermore, the CoFe LDH $\| \mathrm{V}-\mathrm{Ce} / \mathrm{CoFe} \mathrm{LDH}$ cell displays an excellent electrochemical durability with no noticeable decrease at a constant applied potential of $1.65 \mathrm{~V}$ for $60 \mathrm{~h}$ (Fig. 5b). The generated $\mathrm{O}_{2}$ and $\mathrm{H}_{2}$ were further measured quantitatively, and the ratio is close to $1: 2$ (Fig. S26†).

In this work, the exfoliation process leads to an increase of reactive sites in the holes with more dangling bonds and lower coordination numbers. The increasing number of reactive sites from higher specific surface area further leads to the rearrangement of the electronic configuration. Based on the firstprinciple calculation and experimental results, the high HER activity of $\mathrm{V}-\mathrm{Ce} / \mathrm{CoFe} \mathrm{LDH}$ originates from the synergistic effects of large ECSA, oxygen vacancies, and improved electrical conductivity, facilitating fast charge transfer induced by the plasma etching process. $^{29}$ More importantly, phase transformation processes occurred during this treatment (this effect is the first time reported in this work). For the CoFe- and $\mathrm{Ce} /$ CoFe LDH powders, two phases of CoFe $\mathrm{LDH}$ and $\mathrm{CoFe}_{2} \mathrm{O}_{4}$ both exist, and the $\mathrm{CoFe} \mathrm{LDH}$ transforms into $\mathrm{CoFe}_{2} \mathrm{O}_{4}$ with increasing plasma time. For the CoFe $\mathrm{LDH}$ grown on nickel foam, less $\mathrm{CoFe}_{2} \mathrm{O}_{4}$ is possibly formed, but with plasma etching, the $\mathrm{LDH}$ phase is changed to an oxy-hydroxide phase as confirmed by XPS and Raman analysis (Fig. S10c, S11c, and $\mathrm{S} 12 \dagger)$. Both CoFe $\mathrm{LDH}$ and $\mathrm{Ce} / \mathrm{CoFe} \mathrm{LDH}$ tend to increase the lattice strain with increasing plasma etching time, and a larger strain is introduced in the $\mathrm{Ce} / \mathrm{CoFe} \mathrm{LDH}$ as compared to the CoFe LDH. Therefore, it is deduced that the plasma etching strategy not only induces vacancies and defects, but can also induce beneficial phase transformations.

\section{Conclusions}

In summary, we synthesize CoFe LDH with oxygen-vacancy defects by nitrogen plasma etching and demonstrate the resulting material as a highly active HER catalyst in alkaline media. The as-prepared V-CoFe LDH was further modified with Ce doping. It maintains the two-dimensional nanosheet structure but with enlarged ECSA and active sites, and with better electrical conductivity. During the plasma etching, a phase transformation of CoFe $\mathrm{LDH}$ into $\mathrm{CoFe}_{2} \mathrm{O}_{4}$ further improves the electrocatalytic reaction kinetics. The resulting V-Ce/CoFe LDH shows a high activity $\left(73 \mathrm{mV}\right.$ at $10 \mathrm{~mA} \mathrm{~cm} \mathrm{~cm}^{-2}$ ) and a good durability in $1 \mathrm{M} \mathrm{KOH}$ electrolyte. Such CoFe LDH $\| \mathrm{V}-\mathrm{Ce} / \mathrm{CoFe} \mathrm{LDH}$ is capable of overall water splitting with a cell voltage as low as $1.65 \mathrm{~V}$ at $10 \mathrm{~mA} \mathrm{~cm}^{-2}$ and outstanding long-term stability. Our work provides a new strategy to the realization of LDH-based electrocatalysts with excellent HER performances.

\section{Conflicts of interest}

There are no conflicts to declare.

\section{Acknowledgements}

L. W. designed the experiment. S. L. performed the materials synthesis and characterization. J. Z., M. S., Z. M., K. H., and X. L. assisted the experiment. T. N. performed the XRD and 2D XRD mapping experiment and analysed the data. L. W. wrote the manuscript. L. W., T. N., and P. S. corrected the manuscript. P. S. helped to interpret the results. All authors approved the manuscript. We acknowledge the financial support from the National Natural Science Foundations of China (No. 51802320 and 21965024), the start-up funding from Inner Mongolia University (21300-5195102), and the Opening Project of Key Laboratory of Materials Processing and Mold from Zhengzhou University.

\section{Notes and references}

1 L. Han, S. Dong and E. Wang, Adv. Mater., 2016, 28, 92669291.

2 J. Liu, D. Zhu, Y. Zheng, A. Vasileff and S.-Z. Qiao, ACS Catal., 2018, 8, 6707-6732.

3 V. Vij, S. Sultan, A. M. Harzandi, A. Meena, J. N. Tiwari, W.-G. Lee, T. Yoon and K. S. Kim, ACS Catal., 2017, 7, 7196-7225.

4 Z. Chen, X. Duan, W. Wei, S. Wang and B.-J. Ni, J. Mater. Chem. A, 2019, 7, 14971-15005.

5 J. F. Callejas, C. G. Read, C. W. Roske, N. S. Lewis and R. E. Schaak, Chem. Mater., 2016, 28, 6017-6044.

6 R. Yang, Y. Zhou, Y. Xing, D. Li, D. Jiang, M. Chen, W. Shi and S. Yuan, Appl. Catal., B, 2019, 253, 131-139.

7 Y. Li, M. Lu, Y. Wu, H. Xu, J. Gao and J. Yao, Adv. Mater. Interfaces, 2019, 6, 1900290.

8 G. Rajeshkhanna, T. I. Singh, N. H. Kim and J. H. Lee, ACS Appl. Mater. Interfaces, 2018, 10, 42453-42468.

9 T. Zhang, L. Hang, Y. Sun, D. Men, X. Li, L. Wen, X. Lyu and Y. Li, Nanoscale Horiz., 2019, 4, 1132-1138.

10 P. F. Liu, S. Yang, B. Zhang and H. G. Yang, ACS Appl. Mater. Interfaces, 2016, 8, 34474-34481.

11 X. Wang, Y. Yang, L. Diao, Y. Tang, F. He, E. Liu, C. He, C. Shi, J. Li, J. Sha, S. Ji, P. Zhang, L. Ma and N. Zhao, ACS Appl. Mater. Interfaces, 2018, 10, 35145-35153.

12 H. Xu, B. Wang, C. Shan, P. Xi, W. Liu and Y. Tang, ACS Appl. Mater. Interfaces, 2018, 10, 6336-6345.

13 M. Asnavandi, Y. Yin, Y. Li, C. Sun and C. Zhao, ACS Energy Lett., 2018, 3, 1515-1520.

14 L. Xu, Q. Jiang, Z. Xiao, X. Li, J. Huo, S. Wang and L. Dai, Angew. Chem., Int. Ed., 2016, 55, 5277-5281.

15 Y. Wang, Y. Zhang, Z. Liu, C. Xie, S. Feng, D. Liu, M. Shao and S. Wang, Angew. Chem., Int. Ed., 2017, 56, 5867-5871.

16 R. Liu, Y. Wang, D. Liu, Y. Zou and S. Wang, Adv. Mater., 2017, 29, 1701546.

17 Y. Wang, C. Xie, Z. Zhang, D. Liu, R. Chen and S. Wang, Adv. Funct. Mater., 2018, 28, 1703363.

18 S. Niu, W.-J. Jiang, Z. Wei, T. Tang, J. Ma, J.-S. Hu and L.-J. Wan, J. Am. Chem. Soc., 2019, 141, 7005-7013.

19 A. E. Arrassi, Z. Liu, M. V. Evers, N. Blanc, G. Bendt, S. Saddeler, D. Tetzlaff, D. Pohl, C. Damm, S. Schulz and K. Tschulik, J. Am. Chem. Soc., 2019, 141, 9197-9201.

$20 \mathrm{~V}$. Mote, Y. Purushotham and B. Dole, Journal of Theoretical and Applied Physics, 2012, 6, 6. 
21 D. Zhou, S. Wang, Y. Jia, X. Xiong, H. Yang, S. Liu, J. Tang, J. Zhang, D. Liu, L. Zheng, Y. Kuang, X. Sun and B. Liu, Angew. Chem., 2019, 131, 746-750.

22 H. B. Tao, L. Fang, J. Chen, H. B. Yang, J. Gao, J. Miao, S. Chen and B. Liu, J. Am. Chem. Soc., 2016, 138, 9978-9985.

23 A. Tamilselvan, S. Balakumar, M. Sakar, C. Nayek, P. Murugavel and K. Saravana Kumar, Dalton Trans., 2014, 43, 5731-5738.

24 V. R. R. Cunha, P. A. D. Petersen, M. B. Goncalves, H. M. Petrilli, C. Taviot-Gueho, F. Leroux, M. L. A. Temperini and V. R. L. Constantino, Chem. Mater., 2012, 24, 1415-1425.
25 G. Tsoukleri, J. Parthenios, K. Papagelis, R. Jalil, A. C. Ferrari, A. K. Geim, K. S. Novoselov and C. Galiotis, Small, 2009, 5, 2397-2402.

26 R. Yang, Y. Zhou, Y. Xing, D. Li, D. Jiang, M. Chen, W. Shi and S. Yuan, Appl. Catal., B, 2019, 253, 131.

27 J. Huang, Q. Shang, Y. Huang, F. Tang, Q. Zhang, Q. Liu, S. Jiang, F. Hu, W. Liu, Y. Luo, T. Yao, Y. Jiang, Z. Pan, Z. Sun and S. Wei, Angew. Chem., Int. Ed., 2016, 55, 21372141.

28 Q. Zhang, S. Zhang, Y. Tian and S. Zhan, ACS Sustainable Chem. Eng., 2018, 6, 15411-15418.

29 Z. Yuan, S.-M. Bak, P. Li, Y. Jia, L. Zheng, Y. Zhou, L. Bai, E. Hu, X.-Q. Yang, Z. Cai, Y. Sun and X. Sun, ACS Energy Lett., 2019, 4, 1412-1418. 